\title{
Transbronchial decompression of emphysematous bullae: a new therapeutic approach
}

\author{
P. Bhattacharyya, D. Sarkar, S. Nag, S. Ghosh and S. Roychoudhury
}

ABSTRACT: Bullae are common accompaniments of chronic obstructive pulmonary disease especially emphysema. They contribute to increased lung volume and worsen the mechanical disadvantage of the inspiratory muscles by increasing the residual volume (RV) and RV/total lung capacity ratio. Thus effective decompression of a large bulla or bullae is thus important to improve the lung function of affected patients and also to provide symptomatic relief. Surgery and thoracoscopy are two commonly performed procedures used to treat bullae. Although bronchoscopic lung volume reduction has been successfully accomplished for emphysema, isolated decompression of bullae bronchoscopically has not been tried to date.

A large emphysematous bulla in the left lower lobe of a surgically unfit patient was bronchoscopically punctured with a transbronchial aspiration needle; the position of the needle inside the bulla was confirmed and the air from the bulla was aspirated slowly to allow collapse. Finally, some autologous blood was instilled into the bulla before the needle was withdrawn.

The patient had immediate and sustained symptomatic relief with significant improvement in lung function.

Bronchoscopic transbronchial decompression of emphysematous bullae can be an effective therapeutic option and warrants further investigation.

KEYWORDS: Bronchoscopic lung volume reduction, emphysematous bulla, fibreoptic bronchoscopy, lung volume reduction surgery

A 50-yr-old male nonsmoker, presented with incapacitating shortness of breath, cough, fever and increased expectoration of mucoid sputum for 2 weeks. The patients had been suffering from progressive shortness of breath with episodic exacerbations associated with cough, expectoration and occasional history of wheeze over the previous 15 yrs. The patient had stopped smoking 7 yrs earlier, prior to which he had smoked heavily for $>20$ yrs.

On examination the patient was poorly nourished (body mass index $16.75 \mathrm{~kg} \cdot \mathrm{m}^{-2}$ ) and grossly dyspnoic (orthopnoic on supplemental oxygen and incapable of getting out of bed), with a cardiac frequency of $130 \mathrm{bpm}$ and blood pressure of $120 / 80 \mathrm{mmHg}$. The chest was emphysematous, however, there was no clubbing, raised jugular venous pressure or pedal oedema, but the accessory muscles of inspiration were working vigorously. The chest bellow was bilaterally symmetrical with reduced expansion $( \pm 1 \mathrm{~cm})$ and obliteration of the liver dullness. Breath sounds were grossly diminished on both sides, especially so on the left base. All other systems were essentially normal on examination.

The laboratory investigations revealed haemoglobin of $11.20 \%$, total leukocyte count was 9,800 per $\mathrm{mm}^{3}$ with neutrophils and lymphocytes consisting 77 and $20 \%$, respectively, and an erythrocyte sedimentation rate of $62 \mathrm{~mm}$. Except initial hypokalaemia $\left(3.2 \mathrm{mEq} \cdot \mathrm{L}^{-1}\right)$, serum electrolytes, blood urea nitrogen, creatinine and liver-function test results were all normal. The sputum did not reveal any acid-fast bacilli on three occasions but sputum culture showed growth of Klebsiella species sensitive to quinolones and aminoglycosides. There was sinus tachycardia on electrocardiography and the chest radiograph (postero-anterior view) showed an emphysematous chest with bilateral scars (in the upper zones) and multiple bullae especially on the left side. The diaphragm was depressed to such an extent that its contour was no longer visible (fig. 1). The computed tomographic plates confirmed multiple bullae in both lungs with one large bulla occupying the anterior aspect of the
AFFILIATIONS

Institute of Pulmocare and Research, Kolkata, India.

CORRESPONDENCE

P. Bhattacharyya

Institute of Pulmocare and Research

CB-16

Sector I

Salt Lake

Kolkata 700064

India

Fax: 913323202729

E-mail: parthachest@yahoo.com

Received:

February 282006

Accepted after revision:

December 222006

STATEMENT OF INTEREST

None declared.

European Respiratory Journal Print ISSN 0903-1936 Online ISSN 1399-3003 


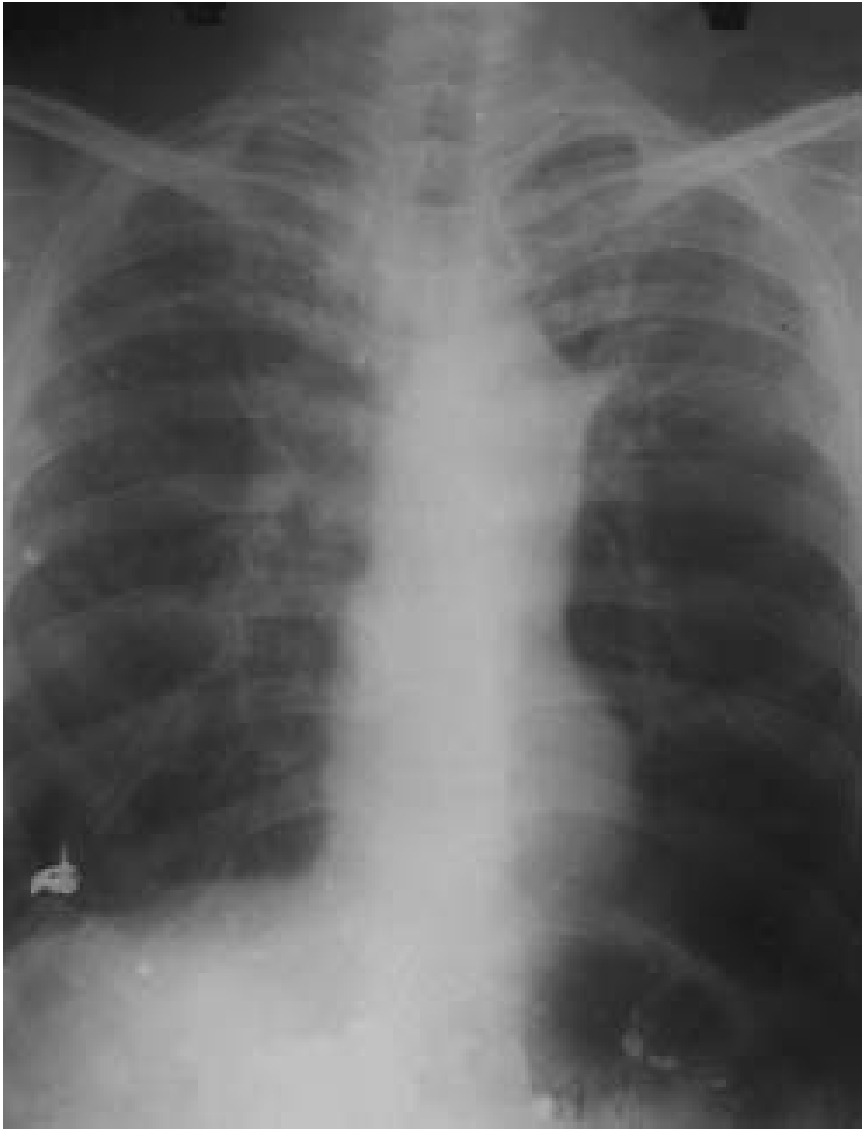

FIGURE 1. Chest radiograph showing bilateral bullous changes with the left side of the diaphragm pushed down and not visible on the radiograph.

left upper lobe and another in the left lower lobe (fig. 2). The initial arterial blood gas (ABG) revealed an arterial carbon dioxide tension of $7.95 \mathrm{kPa}$ and arterial oxygen tension of $9.04 \mathrm{kPa}$ on supplemental oxygen (inspiratory oxygen fraction of $28 \%$ ), with a $\mathrm{pH}$ of 7.4 and bicarbonate of $26 \mathrm{mmol} \cdot \mathrm{L}^{-1}$.

The patient was initially too ill to perform spirometry, but once estabilished with conservative medical management had a forced expiratory volume in one second (FEV1) of $0.68 \mathrm{~L}$ and a forced vital capacity of $1.24 \mathrm{~L}$. A cardiothoracic surgical team was consulted and the patient was declared very high risk for a surgical venture, therefore, an alternative new procedure with less risk was considered.

\section{METHOD}

The proposed procedure was discussed in detail with the patient and written consent, explaining all the possible risks involved, was obtained. The effect of both conservative treatment and the poor surgical prospect was also elaborated. Routine haematological and biochemical (glucose, renal function and liver function) tests were within normal limits and the following morning the patient was prepared with $0.25 \mathrm{mg}$ of alprazolam, sublingually, and $4 \%$ xylocaine nebulisation (total of $8 \mathrm{~mL}$ ) following an overnight fast. As a precaution an intercostal tube drainage system, for inadvertent pneumothorax or bronchopleural fistula, and a cardiothoracic team were kept on standby.
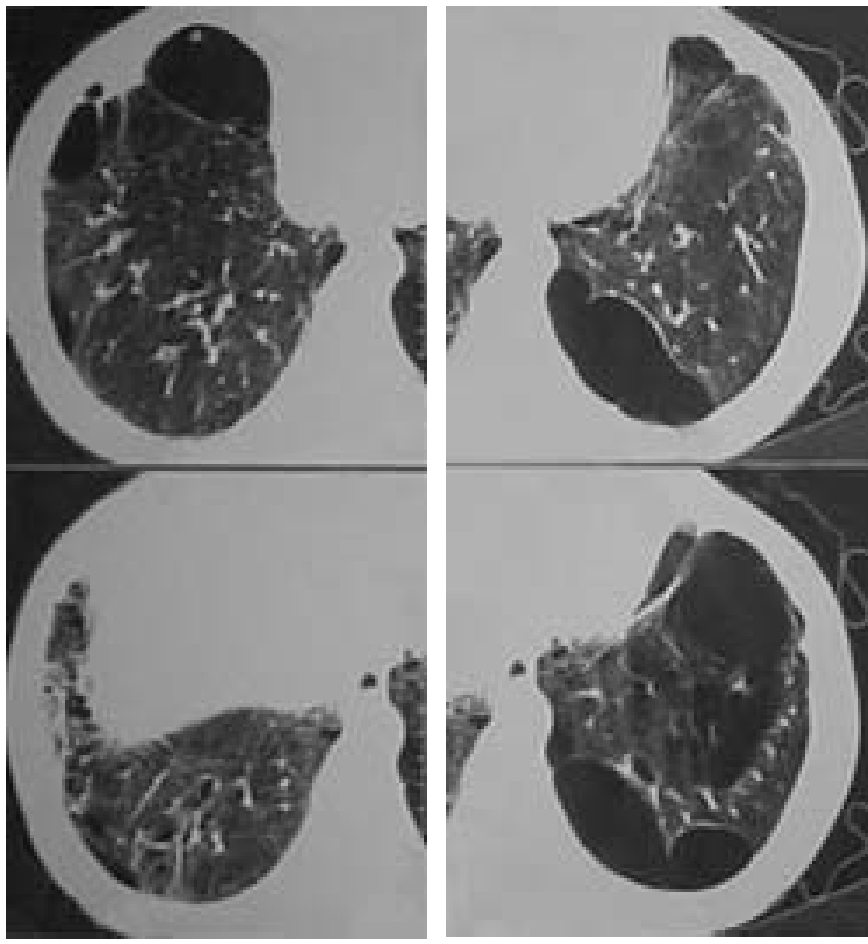

FIGURE 2. High resolution computed tomogrographic image of the thorax showing multiple cystic air spaces in both lungs and left sided pneumothorax.

Fibreoptic bronchoscopy was performed with a Pentax 18P fibrescope (Pentax, Tokyo, Japan) with local instillation of $2 \%$ xylocaine as needed and continuous oxygen supplementation through a nasal cannula placed in the mouth. The tip of the scope was placed deep in the left lower lobe at the anterior basal segment (the site of the biggest bulla) and a 21-gauge transbronchial needle aspiration needle (BARD/Mill-Rose Labs, Billerica, MA, USA) was then passed through the working channel of the bronchoscope. Once the distal hub of the needle was out of the tip of the scope, the needle was ejected and pushed cautiously to pierce the wall of the bulla. A feeling of decreased resistance and an expiratory bubbling of air inside the partially filled waster syringe (fitted to the external proximal hub of the needle and held vertically) confirmed the needle to be within the bullous space. Subsequently, the syringe was removed and a three-way connector attached to the proximal hub in order to aspirate air from the pierced bulla. The air was slowly aspirated and expelled to the atmosphere through an underwater drainage system via the three-way connector. The patient was constantly monitored for pulse, ABGs, blood pressure, electrocardiograph changes and for any cough, pain or physical discomfort. The procedure was uneventful. After aspiration of $\sim 500 \mathrm{~mL}$ of air from the bulla and instillation of $\sim 5 \mathrm{~mL}$ of autologous blood into the bullous space, the needle was removed.

\section{RESULTS}

The patient cooperated fully and expressed ease of breathing and comfort during the procedure. The immediate postprocedure portable chest radiograph showed a significant reduction in air shadows in the base of the left lung and the left dome of the diaphragm was now more elevated than before 
(fig. 3). The post-procedure FEV1 (performed 2 days after the procedure) had improved to $1.20 \mathrm{~L}$ compared with the previous $0.68 \mathrm{~L}$. Three days after the procedure, with proper rehabilitation advice, the patient was discharged in a much improved state (able to go to the toilet alone and walk around the ward freely).

During the 48-month follow-up, the patient had three small infective exacerbations and developed pulmonary tuberculosis, all of which were all successfully treated. The patient is doing reasonably well with mild restriction of activities and has been performing his office job satisfactorily. The latest FEV1 (February, 2006) was $1.27 \mathrm{~L}$ and there has been no significant radiological deterioration of the bullae affected area.

\section{DISCUSSION}

Bullae are large nonventilated air-containing intrapulmonary spaces of $>1 \mathrm{~cm}$ in diameter; their walls formed by compressed and attenuated lung tissue. Emphysema is commonly association with bullae [1]. Bullae impart mechanical impediment to the ventilation of adjacent lung parenchyma and also impair the functioning of the inspiratory muscles, especially the diaphragm. These effects alone may cause symptoms even without the further complication of rupture, infection, etc.

The current medical management of bullae has limited scope, and surgery is the preferred option for large emphysematous bulla; surgical resection has been found to improve symptoms, exercise tolerance and respiratory reserve [2,3]. A wide variety of surgical procedures have been proposed in the management

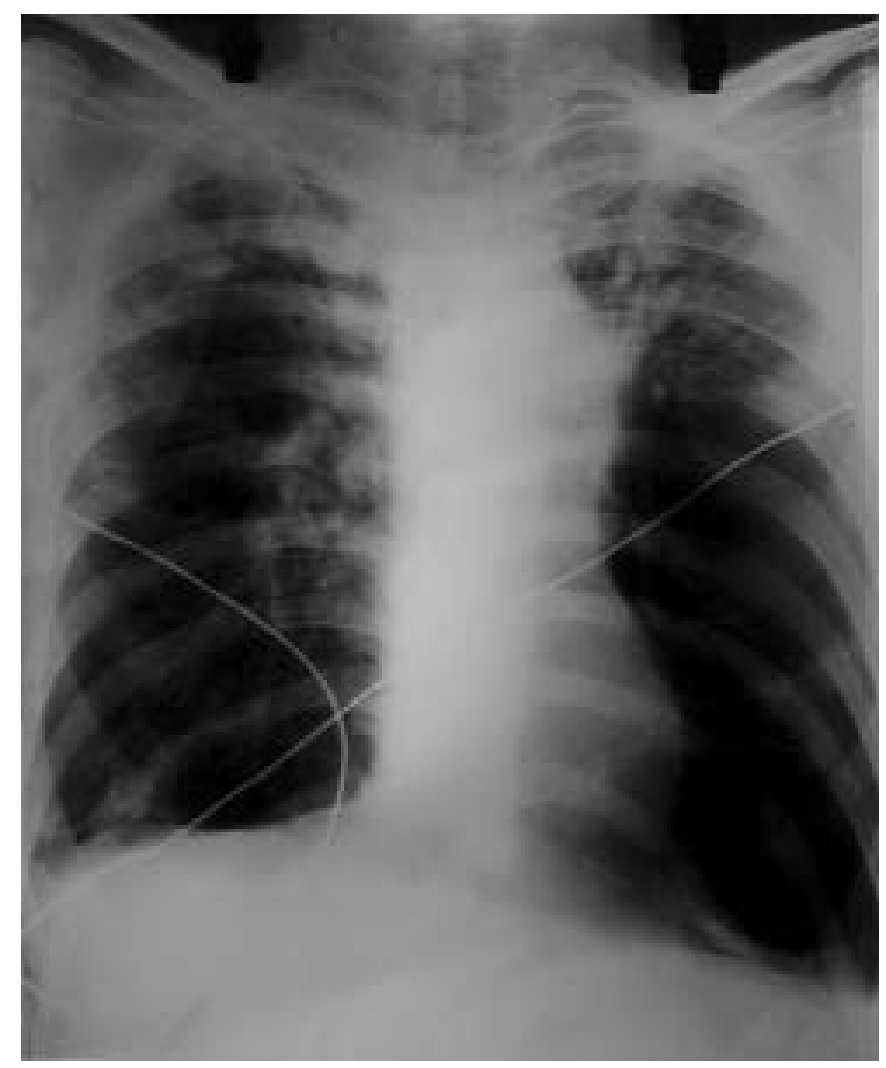

FIGURE 3. Chest radiograph showing partial volume reduction of the left lower lobe bullae with the lower left border of the diaphragm now visible. of bullous emphysema, such as: intra-cavity drainage [4]; local excision of the bullae [5]; placation [6]; stapler resection [7]; and lobectomy [8]. With the increasing interest in lung-volume reduction for emphysema and the subsequent National Emphysema Treatment Trial trial [9], there has been a surge of efforts aimed at nonsurgical lung-volume reduction. Recent thoracoscopic treatment of bullous emphysema of varying severity was associated with universal functional improvement; however, significant post-operative morbidity and a prolonged hospital stay were common problems [10]. Thoracoscopic carbon dioxide laser-ablation treatment of emphysematous bullae has been shown to result in an impressive improvement in physiological paramenters [11]. External drainage of bulla via a catheter has been attempted in emphysematous bullae; out of 31 patients there was one failure and two operative deaths [12].

Bronchoscopic lung volume reduction (BLVR) has recently shown promise. BLVR has been tried with either the placement of a valve (or similar device) in the segmental bronchi to produce distal atelactasis, or by inducing collapse or fibrosis via selective chemical treatment of the affected lung areas. The placement of a valve prosthesis can be performed bronchoscopically in human lungs [13], but although this has been proven safe and effective, some complications have been noted [14, 15]. Recently, an umbrella-shaped device (Spiration, Redmond, WA, USA), consisting of a polyurethane membrane on a nitinol frame, has been tried and shows promise [16]. Furthermore, LAUSBERG et al. [17] recently reported a technique involving fenestration of the segmental bronchi by radiofrequency probes (Exhale RF Probe; Bronchus Technologies, Mountain View, CA, USA) in order to enhance the expiratory airflow in excised emphysematous lungs. The bronchopulmonary fenestrations were maintained by the placement of uncovered coronary stents and the study reported an impressive improvement in the mean FEV1 by $83 \%$.

Injection of a fibrin-based sealant into the tissue-engineered emphysematous area of lungs in a sheep model has been performed successfully [18]. This process has been further modified with bronchoscopic instillation of fibrin hydrogel and thrombin in six sheep with papaine-induced emphysema, following enzyme (trypsin) treatment of the targeted area [19].

None of the above-mentioned lung-volume reduction experiments or trials selectively targeted the issue of bullae as in the current study. Moreover, in the BLVR techniques, a small but definite volume of functional lung tissue is likely to be compromised along with emphysematous areas in the patients with COPD. In this regard, the current technique is unique since it only targets the bullae-affected lung, does not compromise existing lung function and allows the compressed but potentially functional lung parenchyma to contribute to post-procedure lung function. A small volume of autologous blood was instilled inside the bulla to induce fibrosis of the bulla wall and to prevent re-expansion of the decompressed bulla. The procedure appears successful as subsequent chest radiographs show no increment in the size of the treated bulla after $>4.5$ yrs follow-up. The transbronchial decompression of bullae in this particular patient was possible with little technical help or complication. However, there was an obvious risk of pneumothorax or bronchopleural fistula which was avoided by remaining quite proximal in the bronchial tree. The 
risk of such complications would make this procedure unsuitable for peripheral bullae. This technique may also prove effective in single large bulla but there may be a chance of re-expansion oedema developing due to the sudden decompression of the lung parenchyma compressed by the bulla. However, with the adaptation of appropriate precautions, some technical modifications, the development of a better drainage system (with manufacture of a specialised needle) and the help of a C-arm image intensifier, this procedure can be made far more effective and safe. Further performance of the same procedure can only validate its role as a therapeutic venture, standardise the technique, and help identify the candidates who would benefit most.

\section{REFERENCES}

1 Reid L. The pathology of emphysema. Lloyd-Luke, London, 1967; pp. 211-240.

2 FitzGerald MX, Keelan PJ, Cugell DW, Gaensler EA. Long term results of surgery for bullous emphysema. J Thorac Cardiovasc Surg 1974; 68: 566-587.

3 Connolly JE, Wilson A. The current status of surgery for bullous emphysema. J Thorac Cardiovasc Surg 1989; 97: 351-361.

4 Venn GE, Williams PR, Goldstraw P. Intracavitary drainage for bullous emphysematous lung disease: experience with the Brompton technique. Thorax 1988; 43: 998-1002.

5 Wesley JR, Macleod WM, Mullard KS. Evaluation and surgery of bullous emphysema. J Thorac Cardiovasc Surg 1972; 63: 945-955.

6 Benfield JR, Cree EM, Pellett JR, Barbee R, Mendenhall JT, Hickey RC. Current approach to the surgical management of emphysema. Arch Surg 1966; 93: 59-70.

7 Deslauriers J, Leblanc P, McClish A. Bullous and bleb disease of the lung. In: Shields TW, ed. General Thoracic Surgery. Philadelphia, Lea and Febiger, 1989; p. 727.

8 Woo-Ming M, Chapel LH, Belcher JR. The results of surgical treatment of large air cysts of the lung. $\mathrm{Br} J$ Dis Chest 1963; 57: 79-85.

9 Fishman A, Martinez F, Naunheim K, et al. A randomized trial comparing lung volume-reduction surgery with medical therapy for severe emphysema. $N$ Engl J Med 2003; 348: 2059-2073.

10 De Giacomo T, Venuta F, Rendina Erino A, et al. Videoassisted thoracoscopic treatment of giant bullae associated with emphysema. Eur J Cardiothorac Surg 1999; 15: 753-757.

11 Brenner M, Kayaleh KA, Milne EN, et al. Thoracoscopic laser ablation of pulmonary bullae: radiographic selection and treatment response. J Thorac Cardiovasc Surg 1994; 107: 883-890.

12 Macarthur AM, Fountain SW. Intracavity suction and drainage in the treatment of emphysematous bullae. Thorax 1977; 32: 668-672.

13 Snell GI, Holsworth L, Borrill ZL, et al. The potential for bronchoscopic lung volume reduction using bronchial prostheses: a pilot study. Chest 2003; 124: 1073-1080.

14 Venuta F, De Giacomo T, Rendina EA, et al. Bronchoscopic lung-volume reduction with one-way valves in patients with heterogenous emphysema. Ann Thorac Surg 2005; 79: 411-416.

15 Toma TP, Hopkinson NS, Hillier J, et al. Bronchoscopic volume reduction with valve implants in patients with severe emphysema. Lancet 2003; 361: 931-933.

16 Dillard DH, Gonzalez X, DeVore LJ, et al. Evaluation of a novel intra-bronchial valve to produce lung volume reduction. Presented at the World Congress of Bronchology, June 16-19, 2002, Boston, MA, USA. Available at: www.spirationinc.com/downloads/evaluation_novel_intrabronchial_valve.pdf. Date last accessed: January 12, 2007.

17 Lausberg HF, Chino K, Patterson GA, Meyers BF, Toeniskoetter PD, Cooper JD. Bronchial fenestration improves expiratory flow in emphysematous human lungs. Ann Thorac Surg 2003; 75: 393-397.

18 Ingenito EP, Reilly JJ, Mentzer SJ, et al. Bronchoscopic volume reduction: a safe and effective alternative to surgical therapy for emphysema. Am J Respir Crit Care Med 2001; 164: 295-301.

19 Ingenito EP, Berger RL, Henderson AC, Reilly JJ, Tsai L, Hoffman A. Bronchoscopic lung volume reduction using tissue engineering principles. Am J Respir Crit Care Med 2003; 167: 771-778. 